\title{
Advances in Collaborative Documentation Support for CMIP6
}

EGU 2020

4-8 May 2020

C. Pascoe, D. Hassell, M. Stockhause, M. Greenslade, S. Bartholomew

EGU2020-19636: https://doi.org//0.5 194/egusphere-egu2020-19636 


\section{Earth System Documentation ES-DOC}

The Earth System Documentation (ESDOC) project nurtures an ecosystem of tools \& services to enable the scientific community to better understand and utilise Earth system model data.

ES-DOC describes the entire workflow that created the data.

ES-DOC documentation includes information about the Project and Experiment design, Model formulation and Ensemble and Simulation descriptions.

ES-DOC documentation also links to the DKRZ data citation service.
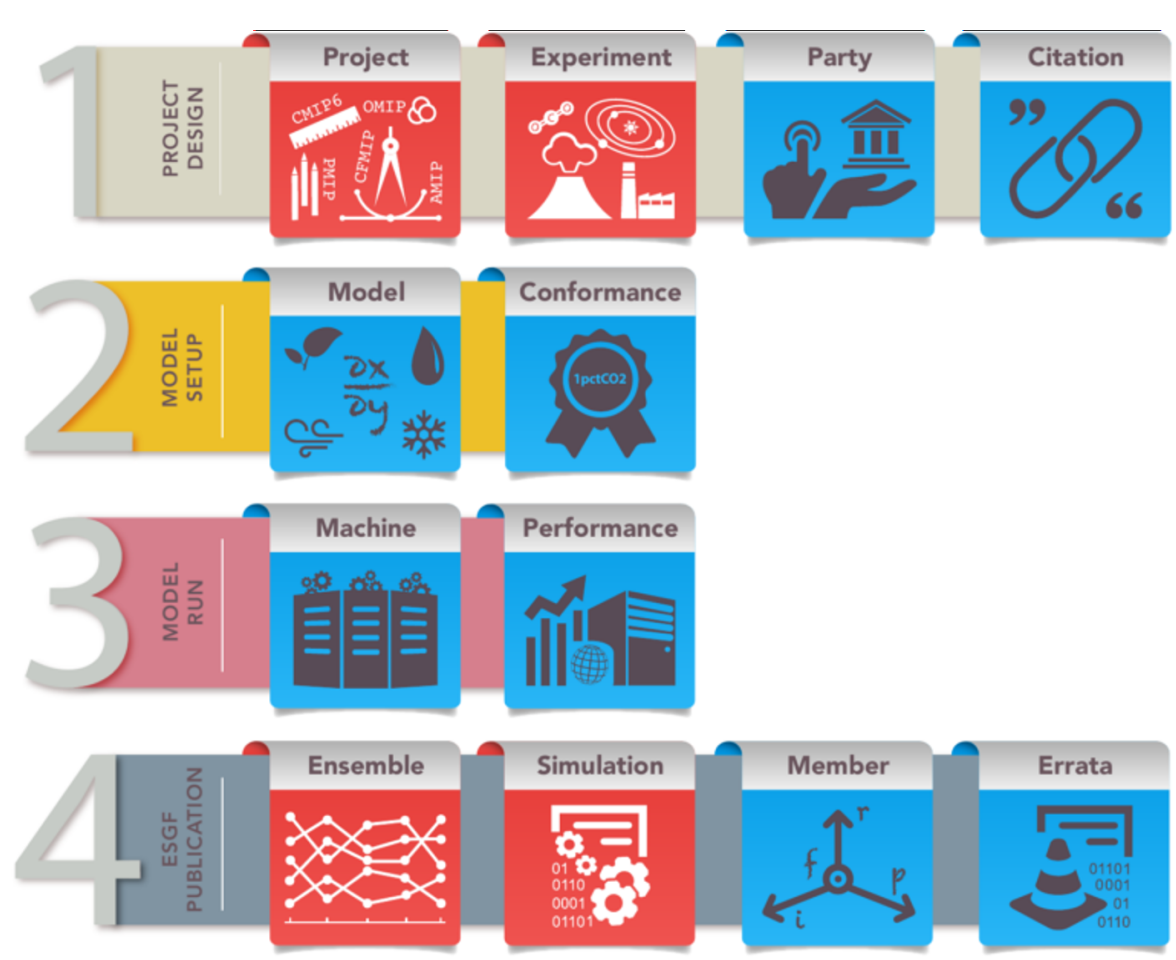

Simulation

Member Errata

Documentation task carried out by:

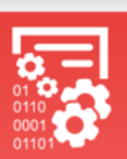

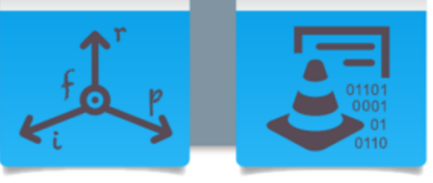

(C) 


\section{ES-DOC CMIP6 Online and Connected}

Modelling groups contributing to the Coupled Model Intercomparison Project (Phase 6) (CMIP6) are using ES-DOC to describe their climate models.

ES-DOC has generated complete structured descriptions of the CMIP6 MIPs and experiments.

ES-DOC auto-generates documentation for every CMIP6 simulation in the Earth System Grid Federation (ESGF).

The ES-DOC information can be accessed via the "further info URL" that is found in every CMIP6 netCDF data file.

The further info URL is a landing page from which all of the CMIP6 documentation relevant to the data may be accessed.

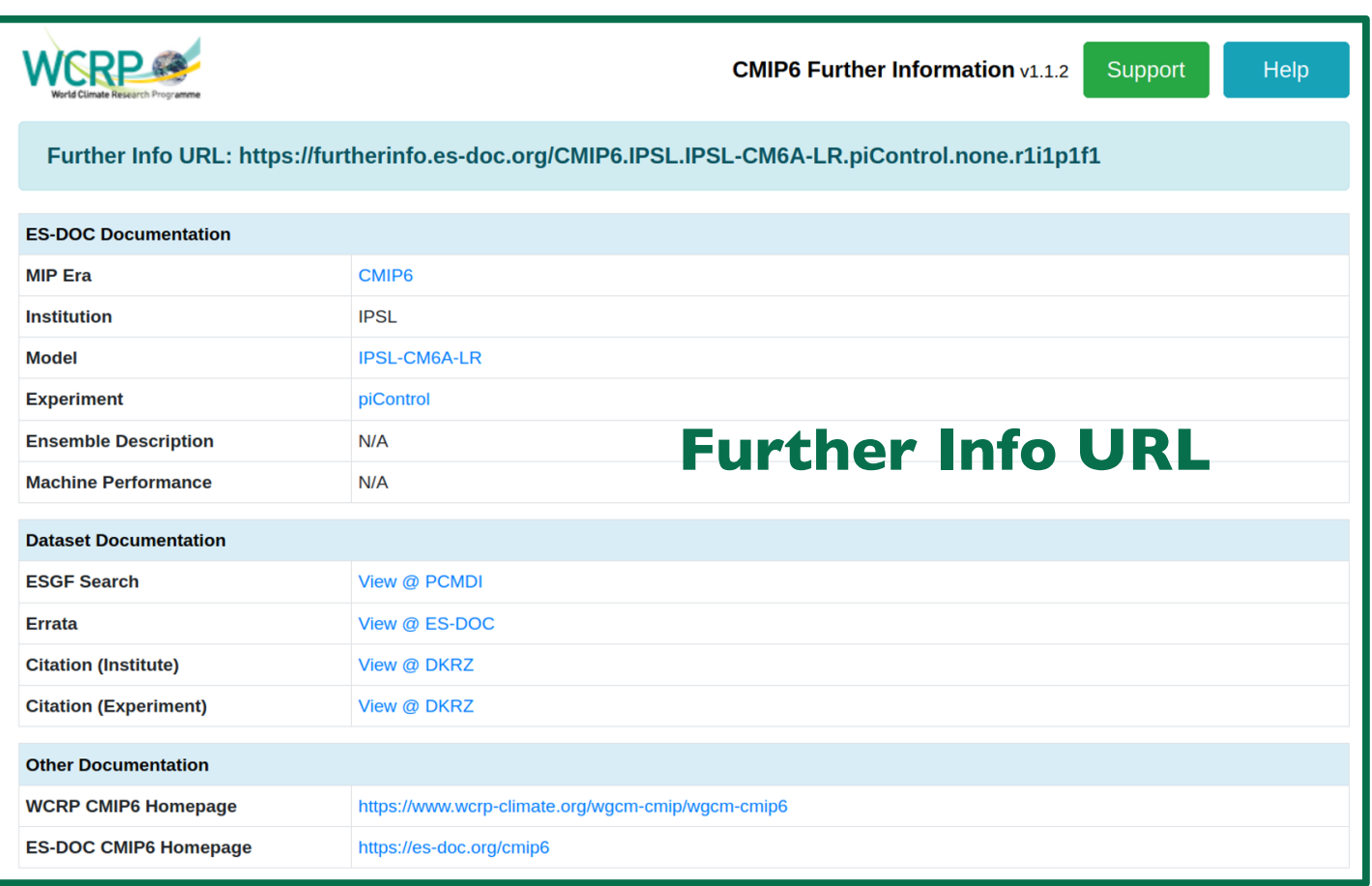

\$ ncdump pr_Amon_piControl_IPSL.IPSL-CM6A-LR-LR_rlilplf1.nc | grep further_info_url : further_info_url = "https://furtherinfo.es-doc.org/CMIP6.IPSI.IPSI-CM6A- 


\section{Further Info URL}

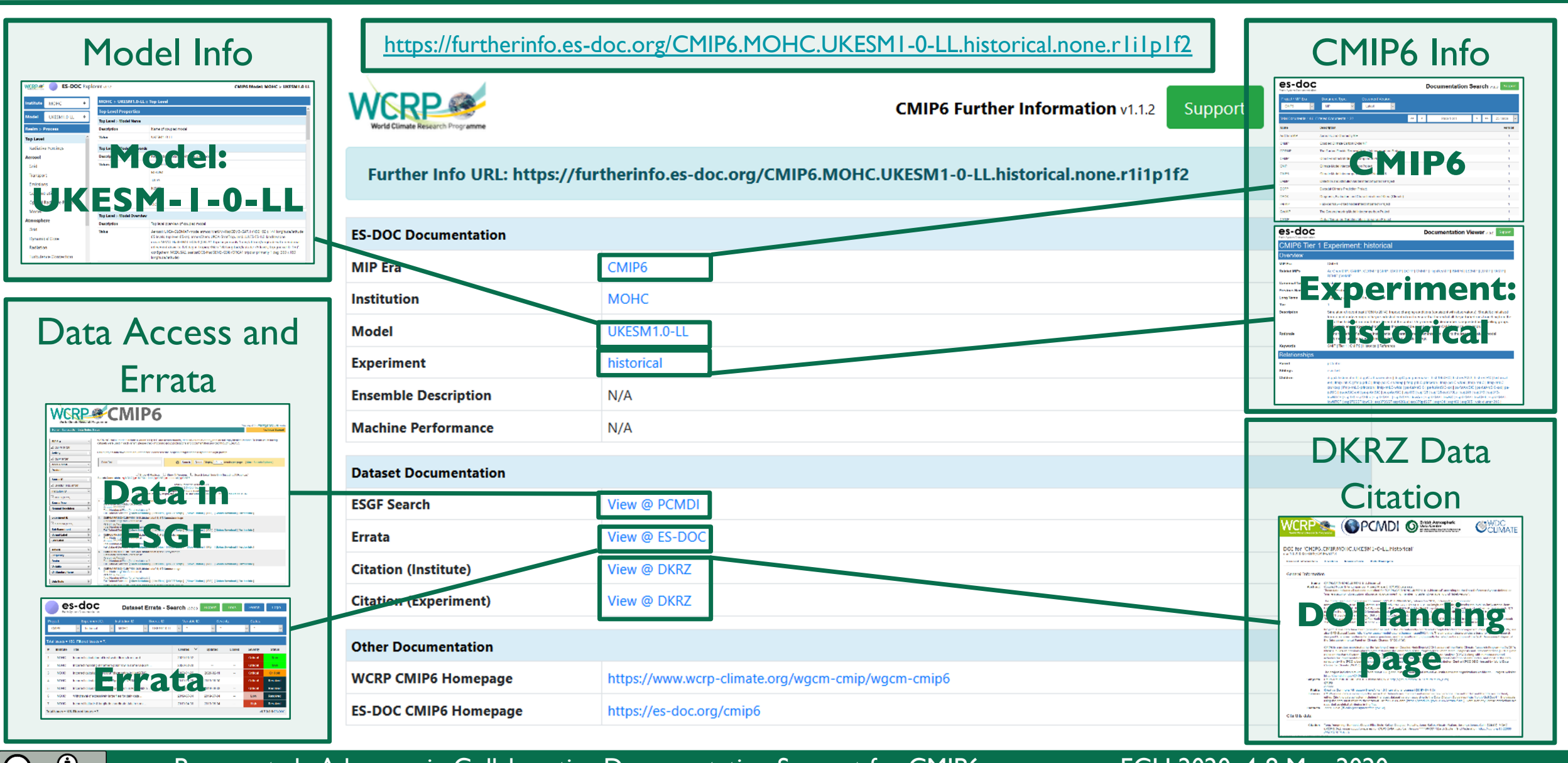




\section{Collecting Structured Documentation}

Structured scientific metadata has an important role in science

communication, however it's

creation and collation exacts a cost

in time, energy and attention.

We look to find a balance between the ease of information collection and the complexity of our

information handling structures.

Modellers are willing to engage in such endeavours where the effort is perceived as being relevant and helpful to their science.

To this end it is important to have tools in place that can be used by scientists to exploit the metadata resources that they so generously provide.
ES-DOC services and the Citation Service depend upon structured documentation provided by the scientific community.

adds content to:

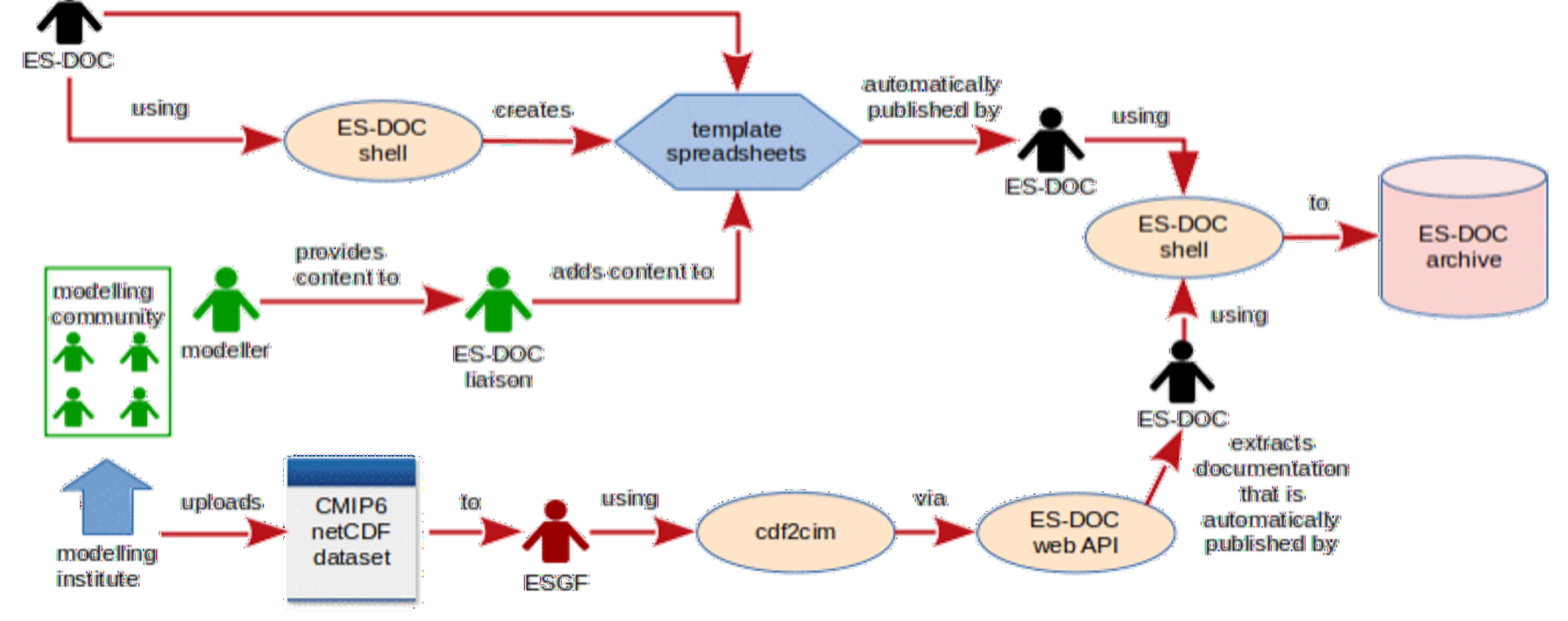




\section{Tools and Services for Understanding Data}

Scientists involved in CMIP6 will find the ES-DOC infrastructure helpful.

Whether they are...

- Highlighting dataset errors.

- Exploring experiment protocols.

- Comparing forcings across ensemble runs.

- Understanding Model Intercomparison Project objectives.

- Reviewing citations.

- Exploring component properties of configured models.

- Visualising inter-model relationships.

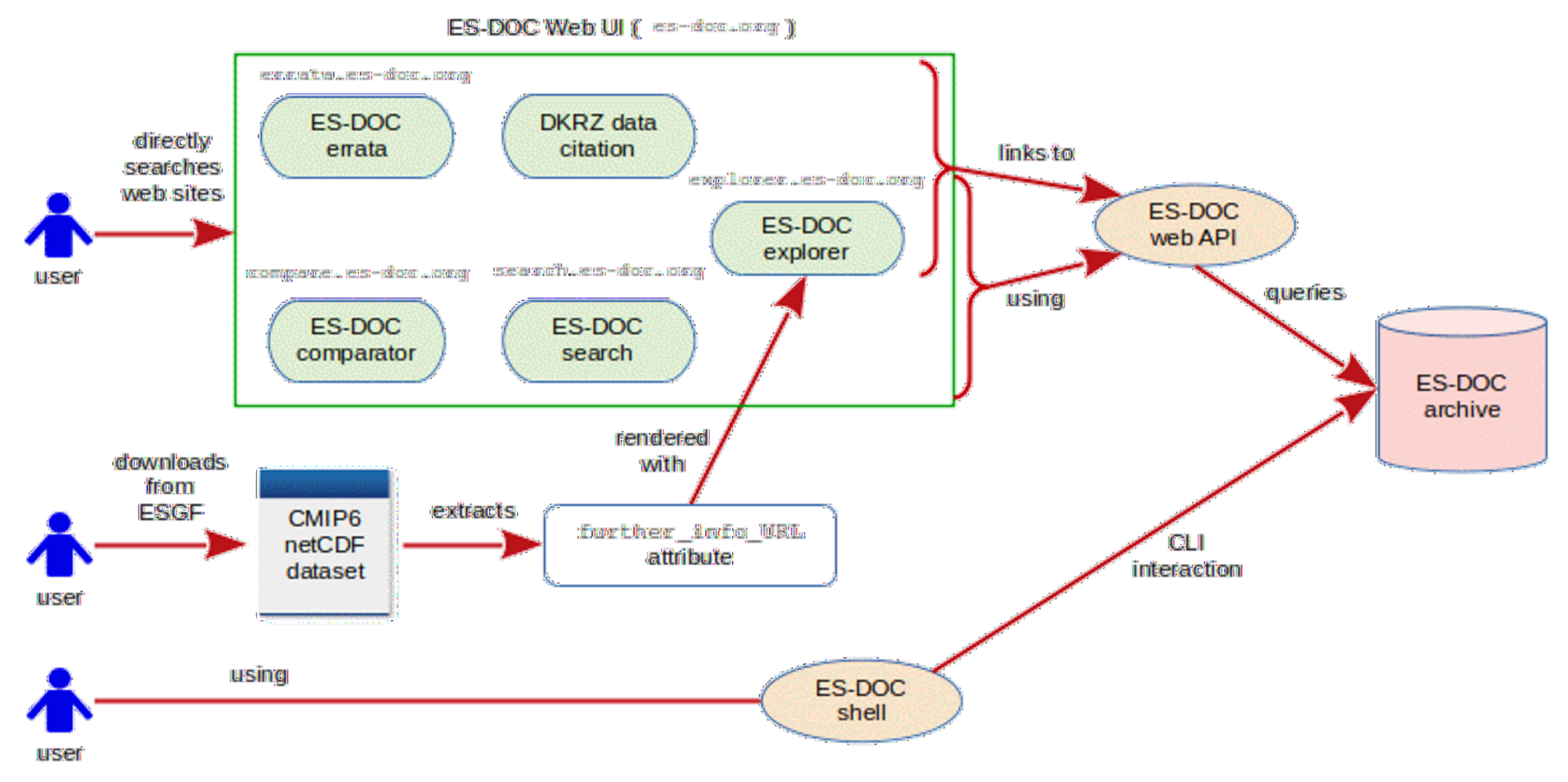


ES-DOC has been operational for CMIP6 since December 2018

- Experiments fully documented by ES-DOC at http://search.es-doc.org

- Modelling groups have started to use the ES-DOC infrastructure to document their model formulations: 10/49 Groups have (partially) published 22/ 30 model descriptions

- Spreadsheets disseminated via GitHub are filled in, returned to GitHub, and then automatically published to http://search.es-doc.org

- This methodology has been designed to be generalisable to the other types of documentation that will be rolled out later in 2020 including:

- Conformance, Machine, performance, Differences between ensemble members

- Simulations are documented by an automated script running as part of the ESGF publisher

- Metadata from 995 institute/model/experiment combinations spanning from at least 2230267 publications have been collected, more arriving all the time.

- These are currently being reduced to $\mathrm{O}(1000)$ unique publishable simulation descriptions

- Further Info URL found in each CMIP6 netCDF files is fully functional, including links to errata citation services 


\section{Status of DKRZ Data Citation Service}

The DKRZ Data Citation Service provides users of CMIP6 data with the formal citation that should accompany any use of the datasets that comprise their analysis.

Data DOI landing pages are also part of the Citation Service, provided by DKRZ.

The data citation information is also available independently through the ESGF Search portal, in the DataCite search and via Google's dataset search.

\section{$99 \%$ of the registered CMIP6 data is citable}

References:

http://bit.ly/CMIP6 DOI Statistics http://bit.ly/CMIP6 Citation Search

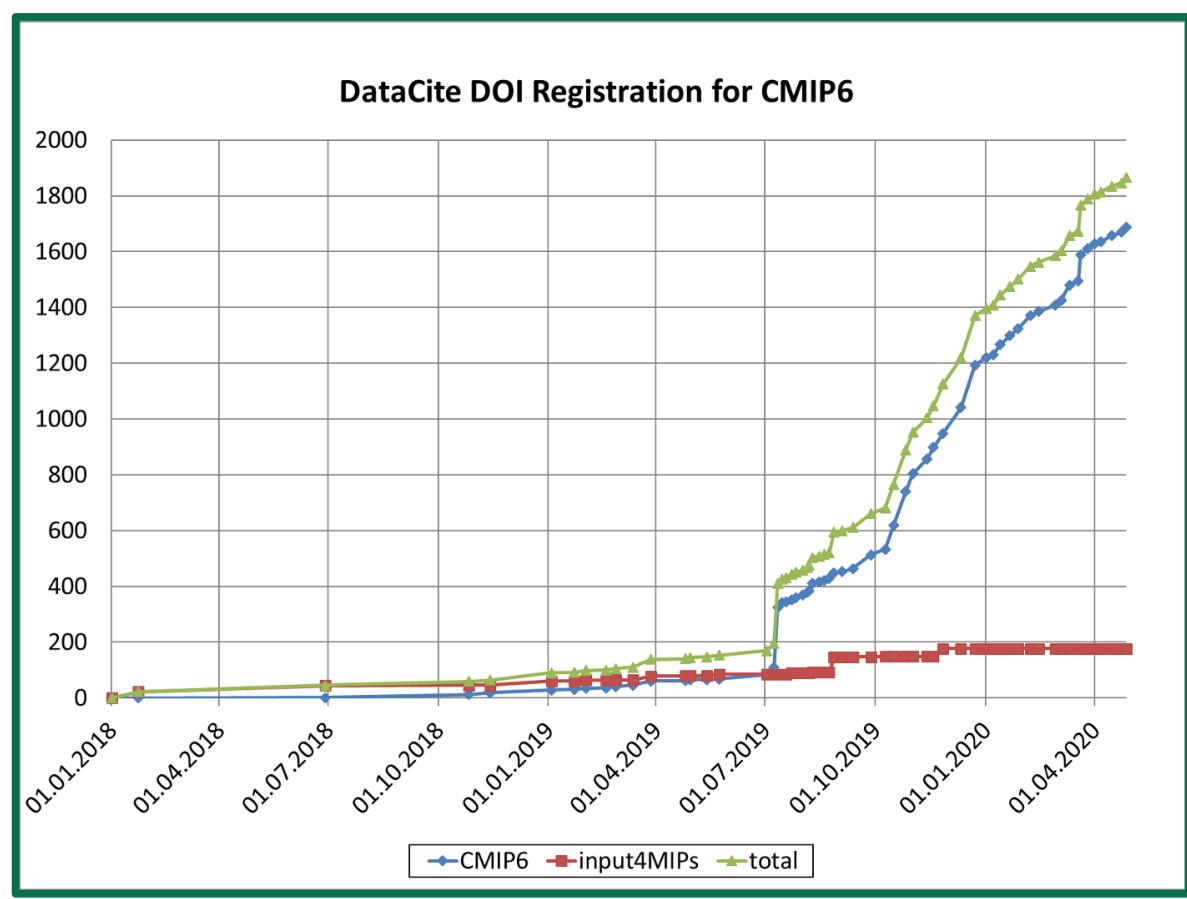

Discover more about the DKRZ citation service on Wednesday in EGU2020-4616: https://doi.org//0.5 194/egusphere-egu2020-46/6 


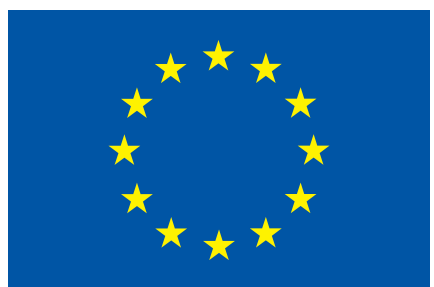

The IS-ENES3 project has received funding from the European Union's Horizon 2020 research and innovation programme under grant agreement $N^{\circ} 824084$

\section{https://is.enes.org}

